\title{
LATTICE MISFIT STRAIN AS ORIGIN FOR BIQUADRATIC INTERLAYER COUPLING
}

\author{
R. JAROSZEWICZ, Z. BĄK AND W. GRUHN \\ Institute of Physics, Pedagogical University \\ Al. Armii Krajowej 13/15, 42-201 Częstochowa, Poland
}

The direct observations of some MBE grown superlattices show large interfacial undulations, which is attributed to the elastic relaxation of tensile layers. Assuming that the interface lateral lattice deformation, due to ionic radii misfit, is given by the periodic solution of the Frenkel-Kontorova model, we calculate the magnetoelastic contribution to the biquadratic magnetic coupling between ferromagnetic layers across the nonmagnetic spacer. We show that due to the correlated modulations of the effective bilinear exchange integral, there arises a biquadratic exchange which favours perpendicular orientations of magnetization in adjacent ferromagnetic layers.

PACS numbers: $75.70 . \mathrm{Cn}, 75.70 . \mathrm{Fr}$

The exchange coupling between ferromagnetic layers separated by a thin nonmagnetic, metallic spacer, is currently an object of intense investigation. Although the dominating mechanism for this exchange interaction has been unambiguously associated with the Ruderman-Kittel-Kasuya-Yoshida (RKKY) interaction [1], the specific mechanisms responsible for the entire coupling are not yet fully understood. The recently discovered $\pi / 2$ coupling between magnetizations on the neighbouring magnetic layers [2] added new interest to the problem. This type of ordering suggests that along with the bilinear coupling of the type $J_{1} m_{1} \cdot m_{2}$ between magnetizations of adjacent layers there is an additional interaction of the form $J_{2}\left(m_{1} \cdot m_{2}\right)^{2}$. Such a term in the effective coupling can be ascribed to the presence of the biquadratic exchange between magnetic moments across the nonmagnetic spacer which for the case $J_{2}>0$ favours the perpendicular orientation of $m_{1}$ and $m_{2}$ (antiferroquadrupolar coupling).

There are a few microscnpical mechanisms which can give rise to the biquadratic coupling in the superlattices, however, the calculations suggest $J_{2}<0$ [3]. The first proof that in superlattices the case $J_{2}>0$ can be realized is due to Slonczewski [4], who has shown that spatial fluctuations of the spacer thickness amounting to one atomic monolayer account phenomenologically for the biquadratic, antiferroquadrupolar coupling. The assumption of Slonczewski [3] that the spacer thickness exerts correlated fluctuations with the monoatomic layer amplitude is far from realistic. However, there is another mechanism which results in 
similar behaviour of the biquadratic exchange as the assumption of Slonczewski. Mismatch of the ionic radii of the adjacent layers constituents may result in a periodic modulation of the ionic separation within spacer layer. In the following we will show that the misfit strains in the interface region of the superlattice, introduced during heteroepitaxial growth, can give rise to the $J_{2}>0$.

Heteroepitaxial growth is always associated with basal-plane lattice mismatch between $A$ and $B$ metallic constituents. The magnitude of lateral strains, arising due to ionic radii mismatch, can be calculated under the assumption that the local distortion is described within the Frenkel-Kontorova model [7]. The Frenkel-Kontorova model consists of a chain of atoms connected by a spring situated in a sinusoidal potential well of the form [7]:

$$
V=\frac{1}{2} \kappa \sum_{n}\left(x_{n+1}-x_{n}-b\right)^{2}-V_{0} \sum_{n} \cos \left(2 \pi x_{n} / a\right),
$$

where $\kappa$ is the force constant of a spring, $V_{0}$ is the strength of the sinusoidal potential well, $b$ and $a$ are the natural periods of the chain and the sinusoidal potential well respectively, and $x_{n}$ is the position of the $n$-th atom. Solving the equation of motion for lattice phonons within perturbation theory in the first order we obtain an expression for the local strain [7]:

$$
\bar{x}_{n} \approx x_{1}^{0}+(n-1) b-\gamma \sin (2 \pi / a)\left[x_{1}^{0}+(n-1) b\right],
$$

where $x_{1}^{0}$ is the zeroth-order position of the chain and $\gamma$ is a constant. In the case of lattice mismatched heteroepitaxy, an initial uniform biaxial coherency strain in the magnetic film is eventually relieved above a critical thickness $t_{\mathrm{c}}$ by the nucleation of misfit dislocation [8]. For $t>s$ (where $s$ is the misfit dislocation spacing) the film experiences an average uniform equilibrium strain. However, for $t<s$ the nonuniform character of the strain field cannot be neglected. In view of Eq. (2) one would expect a sinusoidal modulation of the in-layer lattice distortion and a similar variation of the in-plane stress.

The effect of the lateral strain, modelled within Frenkel-Kontorova model on the conventional, bilinear exchange coupling has been considered in [6]. It was shown that the bilinear exchange integral $J_{1}$ is modulated in space according to the formula [6]:

$$
\begin{aligned}
& J_{1}=J_{0}\left\{1-\frac{1}{4} \Gamma^{2}\left[2-\cos (4 \pi / a)\left[x_{1}^{0}+\left(n_{x}-1\right) b\right]\right.\right. \\
& \left.\left.-\cos (4 \pi / b)\left[y_{1}^{0}+\left(n_{y}-1\right) a\right]\right]\right\},
\end{aligned}
$$

where $a$ and $b$ are the lattice constants of both ferromagnetic and nonmagnetic constituents of the superlattice.

As we can see, the effective bilinear exchange interaction (3) exhibits correlated fluctuations associated with the ionic radii mismatch of both constituents. The distortion of the type (2) exists independently of the fact whether step oscillations postulated by Slonczewski [3] exist or not. In the former case they are superimposed on the step oscillations and since they are relatively small in the first approximation their effect can be neglected. However, in the case of atomically smooth interfaces the fluctuation (2) is dominant. 
To calculate the effect of the lattice spacing oscillation let us follow the approach applied by Slonczewski [3]. We assume that two ferromagnetic (FM) films of thickness $D$ and $D^{\prime}$ are separated by a thin nonmagnetic spacer layer. The magnetizations $\boldsymbol{M}$ and $\boldsymbol{M}^{\prime}$ fluctuate about static values $\boldsymbol{m}_{1}$ and $\boldsymbol{m}_{2}$ in the $x-y$ plane (i.e. the in-layer plane) at angles $\theta(x, z)$ and $\theta^{\prime}\left(x^{\prime}, z^{\prime}\right)$, measured from the common axis. In view of Eq. (3) the Fourier transform of conventional bilinear exchange integral $J(x, y)$ has large amplitude $J_{Q}$ at the wave vector $Q=2 \pi(a-b) / a$ which reflects the periodic modulation (4). However, we must point here that in some cases, due to the vernier effect [11], the effective modulation period can be much longer that $2 \pi / Q$.

The sum of the exchange energies due to the 1D modulation per unit area of the interface is given by [3]:

$$
\begin{aligned}
W_{Q} & =\frac{1}{2 L} \int_{0}^{2 L} \mathrm{~d} x\left\{-J_{Q} \sin Q x \cos \left[\theta(x, 0)-\theta^{\prime}(x, 0)\right]\right. \\
& \left.+A \int_{0}^{D} \mathrm{~d} z\left(\theta_{x}^{2}+\theta_{z}^{2}\right)+A \int_{0}^{D^{\prime}} \mathrm{d} z^{\prime}\left(\theta_{x^{\prime}}^{\prime 2}+\theta_{z^{\prime}}^{\prime 2}\right)\right\} .
\end{aligned}
$$

In the integration (4) the integration range $2 L, D$ and $D^{\prime}$ denote period of the bilinear exchange modulation (3) and the thickness of the neighbouring ferromagnetic layers, respectively. Accounting for the 2D $(x-y)$ modulation of the exchange (3) and repeating the arguments of Slonczewski [3] we obtain that the sum of exchange energies $W_{Q}$ arising due to the oscillation $J_{Q}$ is given by

$$
W_{Q} \approx-J_{Q}^{2}\left[1-\left(m_{1} \cdot m_{2}\right)^{2}\right]
$$

the second term of the expression (5) can be interpreted as biquadratic coupling among magnetizations of adjacent FM layers with positive $\left(J_{2}>0\right)$ exchange integral. In view of the derivation procedure, it is evident that our $J_{2}$ depends on the spacer thickness in the same way as the biquadratic exchange integral of Slonczewski $[3,4]$. Moreover, as we can see from Eq. (5) the magnitude of our $J_{2}$ is proportional to the squared Fourier transform $J_{Q}$ of the integral $J_{1}$. Accounting for the results derived in papers $[6,7]$, we can estimate the relative magnitude of $J_{2}$ as

$$
J_{2} / J_{1}=J_{Q}^{2} / J_{1}=\frac{1}{4} \alpha\left(V_{0} \pi\right)^{2} \hbar^{-2}\left[4 a \alpha \sin ^{2}\left(\frac{\pi b}{a}\right)\right]^{-4} .
$$

Thus, Eq. (6) gives us the magnitude of $J_{2}$ as the function of material constants. Provided that the exchange integral $J_{2}$ is sufficiently large the magnetizations of nearest FM layers are orthogonal.

In coriclusion, we have discussed the influence of elastic effects on the magnetism of strained layers which is relevant to surfaces, films and interfaces. The interface lateral strain is assumed to be given by the periodic solution of the Frenkel-Kontorova model. Provided that the magnetic coupling of ferromagnetic layers across nonmagnetic spacer is given by the RKKY-like bilinear exchange, we prove that the elastic effects associated with misfit strains modify the magnetic interactions. Along with the modifications of bilinear exchange predicted in [6], there appears an effective biquadratic exchange term with positive exchange integral $J_{2}$, 
which favours perpendicular ordering of magnetizations of adjacent ferromagnetic layers. Up to now the fluctuation mechanism of Slonczewski [3] was the only one which resulted in similar effect. The other mechanisms which are giving rise to biquadratic exchange predict ferromagnetic coupling.

\section{Acknowledgments}

Financial support from the Committee for Scientific Research is gratefully acknowledged.

\section{References}

[1] M.A. Ruderman, C. Kittel, Phys. Rev. 96, 99 (1954).

[2] B. Heinrich, J.F. Cochran, M. Kowalevski, J. Kirscher, Z. Celinski, A.S. Arrot, K. Myrtle, Phys. Rev. B 44, 9348 (1991).

[3] J.C. Slonczewski, Phys. Rev. Lett. 67, 3172 (1991).

[4] J.C. Slonczewski, J. Appl. Phys. 73, 5957 (1993).

[5] Z. Bąk, W. Gruhn, J. Alloys Compounds 210, 295 (1994).

[6] Z. Bak, W. Gruhn, R. Jaroszewicz, J. Magn. Magn. Mater. 148, L307 (1995).

[7] J.B. Sokolov, J.E. Sacco, J.F. Weisz, Phys. Rev. Lett. 41, 1561 (1978).

[8] R.C. O'Handley, S.-W. Sun, J. Magn. Magn. Mater. 104-107, 1717 (1992).

[9] Z. Bąk, W. Gruhn, J. Magn. Magn. Mater. 135, L7 (1994).

[10] J. Inoue, J. Magn. Magn. Mater. 136, 233 (1994).

[11] R. Coehoorn, Phys. Rev. B 44, 9331 (1991). 